
\title{
R Reserarch Suare \\ Efficacy and Safety of Luseogliflozin in Patient with Type 2 Diabetes Complicated by Hepatic Dysfunction
}

Hiroaki Seino ( $\nabla$ hiroakiseino@hotmail.co.jp )

Seino Internal Medical Clinic

Research

Keywords: hepatic dysfunction, luseogliflozin, SGLT2 inhibitor, type 2 diabetes mellitus

Posted Date: October 2nd, 2020

DOI: https://doi.org/10.21203/rs.3.rs-84143/v1

License: (c) (i) This work is licensed under a Creative Commons Attribution 4.0 International License.

Read Full License 


\section{Abstract}

Background: Improvements in glycemic control and hepatic function are clinically important goals in the treatment of patients with type 2 diabetes mellitus complicated by hepatic dysfunction. The favorable effects of the sodium-glucose co-transporter inhibitor luseogliflozin on hepatic dysfunction were anticipated for humans. Nevertheless, few clinical studies have confirmed its real-world efficacy on hepatic dysfunction. This trial was conducted to assess the safety and efficacy of luseogliflozin in patients with type 2 diabetes mellitus complicated by hepatic dysfunction.

Methods: This prospective, single-site, single-arm, open-label trial included 55 subjects. Subjects were administered with luseogliflozin and observed for 52 weeks. The primary endpoints were the change and percent change in aspartate aminotransferase (AST), alanine aminotransferase (ALT), gamma-glutamyl transpeptidase ( $\mathrm{Y}-\mathrm{GTP})$, and hemoglobin $\mathrm{A} 1 \mathrm{c}(\mathrm{HbA1c})$ from baseline to week 52. The secondary endpoints included body weight, body mass index (BMI), waist circumference, blood pressure, fasting plasma glucose (FPG), homeostatic model assessment beta (HOMA- $\beta$ ), homeostatic model assessment of insulin resistance (HOMA-IR), ferritin, Mac-2 binding protein (M2-BP), fatty liver index (FLI), fibrosis-4 (FIB-4) index, type IV collagen 7S domain, nonalcoholic fatty liver disease (NAFLD) fibrosis score, highsensitivity C-reactive protein (hs-CRP), and interleukin-6 (IL-6).

Results: AST, ALT, y-GTP, and HbA1c significantly decreased from baseline to week 52. Body weight, BMI, waist circumference, and FPG also significantly decreased. HOMA-IR significantly decreased but HOMA- $\beta$ was unchanged. FLI, ferritin, M2-BP, and NAFLD fibrosis scores significantly decreased whereas the FIB-4 index and type IV collagen 7S domain did not significantly change. The hs-CRP and IL-6 levels did not significantly change.

Conclusion: Luseogliflozin administration in T2DM patients with hepatic dysfunction was well tolerated, improved hepatic function, reduced liver fat, and attenuated liver injury and fibrosis. The present study might help establish a therapeutic approach for T2DM patients with hepatic dysfunction induced by SGLT2 inhibitors.

Trial registration: This study was registered under the University Hospital Medical Information Network Clinical Trial Registry (UMIN-CTR) (No. UMIN000025808) and the Japan Registry of Clinical Trials (No. jRCTs021180017).

\section{Background}

Previous studies have associated type 2 diabetes mellitus (T2DM) with hepatic dysfunction. The association between T2DM and hepatic dysfunction is classified as follows [1, 2]: (1) hepatic dysfunction caused by T2DM (nonalcoholic fatty liver disease (NAFLD), nonalcoholic steatohepatitis (NASH), diabetic hepatopathy, or glycogenic hepatopathy exacerbated by T2DM); (2) T2DM caused by hepatic dysfunction (hepatic diabetes); and (3) hepatic dysfunction simultaneous with T2DM (chronic autoimmune hepatitis and autoimmune hepatic dysfunction). The prevalence of NAFLD in T2DM patients was as high as 50- 
$70 \%[3,4]$. Hepatic dysfunction was a major cause of death in T2DM patients [5-7]. Therefore, improvements in glycemic control and hepatic function are clinically important goals in the treatment of patients with T2DM complicated by hepatic dysfunction.

Novel oral hypoglycemic agents known as sodium glucose co-transporter 2 (SGLT2) inhibitors have been recently launched. They are widely used in T2DM treatment. SGLT2 inhibitors block renal glucose reabsorption, promote urinary glucose excretion, and lower plasma glucose levels in an insulinindependent manner. Hence, there is a reduced risk of induction of hypoglycemia associated with them. The safety of SGLT2 inhibitors in combination with other hypoglycemic agents has been confirmed [8]. Moreover, SGLT2 inhibitors confer cardiovascular protection $[9,10]$, reduce body weight [11], lower mean 24-h glucose levels [12], decrease blood pressure [13], and improve blood lipid factors [14].

Luseogliflozin is a type of SGLT2 inhibitor. It improved NASH in an animal T2DM model [15]. Its favorable effects on hepatic dysfunction were anticipated for humans. Nevertheless, few clinical studies have confirmed its real-world efficacy on hepatic dysfunction. Therefore, the aim of this study was to evaluate luseogliflozin safety and efficacy in patients with T2DM complicated by hepatic dysfunction.

\section{Methods}

\section{Study design}

A single-center, single-arm, open-label, prospective interventional trial was conducted from November 2016 to September 2020. It was registered at the University Hospital Medical Information Network Clinical Trial Registry (UMIN-CTR) which is a nonprofit organization in Japan that meets the requirements of the International Committee of Medical Journal Editors (No. UMIN000025808) and the Japan Registry of Clinical Trials (No. jRCTs021180017). The study protocol was approved by the Institutional Review Board of Seino Internal Medical Clinic in November 2016 according to the Ethical Guidelines for Medical and Health Research Involving Human Subjects issued by the Ministry of Health, Labour and Welfare in Japan. As the Clinical Trials Act was dispensed, the study protocol was reinspected and approved by the Fukushima Medical University Certified Review Board and certification was procured from the Minister of Health, Labour and Welfare in Japan in March 2019. The study was conducted in accordance with the Declaration of Helsinki, the Ethical Guidelines for Medical and Health Research Involving Human Subjects issued by the Ministry of Health, Labour and Welfare in Japan, the Clinical Trials Act, and other current legal regulations in Japan. Written informed consent was obtained from all participants after the study was fully explained to them. To minimize bias, data management and statistical analyses were conducted by third-party entities (DOT World Co. Ltd., Tokyo, Japan; Soiken Inc., Tokyo, Japan).

\section{Patient population}

T2DM patients with hepatic dysfunction were included in the present trial. Patient inclusion criteria were as follows: (1) men and women $\geq 20 \mathrm{y}$ and $\leq 80 \mathrm{y}$ at the time of trial participation consent; (2) poor glycemic control despite diet and exercise therapy or treatment with hypoglycemic agents for $\geq 12 \mathrm{wks}$; 
(3) $\mathrm{HbA} 1 \mathrm{c} \geq 6.5 \%$ and $<9.5 \%$; (4) $\mathrm{BMI} \geq 20 \mathrm{~kg} / \mathrm{m}^{2}$; (5) provision of written informed consent; and (6) hepatic dysfunction (ALT $\geq 31 \mathrm{IU} / \mathrm{L}$ ). Patient exclusion criteria were as follows: (1) type 1 diabetes mellitus; (2) history of severe ketosis, diabetic coma, or precoma; (3) severe pre- or post-surgical infection or serious trauma; (4) severe renal dysfunction (estimated glomerular filtration rate (eGFR) < $30 \mathrm{~mL} / \mathrm{min} / 1.73 \mathrm{~m}^{2}$ ); (5) history of stroke, myocardial infarction, or other serious cardiovascular complications requiring hospitalization $\leq 6$ mo prior to giving trial participation consent; (6) existing use of SGLT2 inhibitor; (7) pregnancy, breastfeeding, possible pregnancy, or intention to become pregnant; (8) history of hypersensitivity to luseogliflozin or any of its components; and (9) any conditions deemed inappropriate by the physicians for trial participation.

\section{Study intervention}

As this study was a single-arm trial, randomization was not conducted. The subjects furnished informed consent and then began to consume $2.5 \mathrm{mg}$ oral luseogliflozin once daily either before or after breakfast. The study intervention was conducted for 52 wks and the subjects were observed at baseline (week 0 ) and at weeks 12, 24, and 52. All enrolled subjects were prohibited from using any SGLT2 inhibitor other than luseogliflozin during the trial. Moreover, enrolled subjects were not permitted to change the type, usage, or dose of any other therapeutic agents such as antiplatelet, antihypertensive, or antidyslipidemia drugs. They were not allowed to alter the type or degree of diet and exercise therapy during the study, provided that these treatments were safely regulated.

\section{Study outcomes}

The primary endpoints comprised the change and percent change in aspartate aminotransferase (AST), alanine aminotransferase (ALT), gamma-glutamyl transpeptidase ( $\mathrm{Y}-\mathrm{GTP})$, and hemoglobin A1c (HbA1c) level from baseline to week 52. The secondary endpoints were as follows: (1) changes in AST, ALT, Y-GTP, and $\mathrm{HbA} 1 \mathrm{c}$ levels from baseline to weeks 12 and 24; (2) changes in fasting plasma glucose (FPG) level, homeostatic model assessment beta (HOMA- $\beta$ ), homeostatic model assessment of insulin resistance (HOMA-IR), body weight, body mass index (BMI), waist circumference, blood pressure, NAFLD fibrosis score, fibrosis-4 (FIB-4) index, and fatty liver index (FLI) from baseline to weeks 12, 24, and 52; (3) changes in type IV collagen 7S domain, ferritin, Mac-2 binding protein (M2-BP), and high-sensitivity Creactive protein (hs-CRP)) level from baseline to weeks 24 and 52; and (4) change in interleukin-6 (IL-6) level from baseline to week 52.

\section{Sample size calculation and statistical analysis}

The target number of enrolled subjects was 50 and was based on the possible number of subjects who could give their consent following daily medical examinations at the Seino Internal Medical Clinic.

The primary and secondary endpoints were evaluated on the Full Analysis Set (FAS) which includes all subjects assigned to a study intervention. However, subjects who did not receive the study agent were excluded from the FAS. Subjects for whom no data related to the efficacy endpoints could be obtained after study agent initiation were also excluded from the FAS. The Per-Protocol Set (PPS) excluded 
subjects from the FAS if they presented with substantial protocol violations such as eligible criteria nonconformance, use of prohibited drugs, and poor adherence to the study agent. The safety analysis included all treated patients. All two-sided tests were performed and $P<0.05$ was considered as statistically significant. Summary statistics (number of subjects, mean, standard deviation, minima, median, and maxima) and changes from baseline were calculated for continuous data. A one-sample $t$ test was conducted to identify the change from baseline. Frequencies and proportions were calculated for the categorical data. SAS version 9.4 (SAS, Cary, NC, USA) was used to perform all statistical analyses.

\section{Results}

\section{Baseline characteristics of the study participants}

Between April 2017 and September 2018, 55 subjects were enrolled in this study and received the intervention (luseogliflozin administration). One subject discontinued the visit to the institution during the study. Five subjects discontinued luseogliflozin use because of adverse events during the study. Hence, 55 subjects were included in both the safety analysis set and the FAS (Fig. 1) and 49 subjects completed the study intervention. The baseline characteristics of the subjects are summarized in Table 1. 
Table 1

Baseline characteristics of subjects

\section{Characteristics}

Age (y)

Gender (male/female)

Height (cm)

Body weight $(\mathrm{kg})$

BMI $\left(\mathrm{kg} / \mathrm{m}^{2}\right)$

Waist circumstance $(\mathrm{cm})$

Duration of diabetes mellitus

Drinking habit

Smoking habit

Cerebrovascular complications

Diabetic complications

Diabetic nephropathy

Diabetic neuropathy

Diabetic retinopathy

Hypertension

Dyslipidemia

Antidiabetic agents

Sulfonylurea

Biguanide

a-Glucosidase inhibitor

Glinide

Thiazolidinedione

DPP-4 inhibitor

SGLT2 inhibitor

GLP-1 receptor agonist mean \pm standard deviation or $n(\%)$

$52.7 \pm 11.4$

38 (69.1) / 17 (30.9)

$165.6 \pm 8.3$

$80.4 \pm 14.9$

$29.2 \pm 4.0$

$98.5 \pm 9.9$

$8.8 \pm 6.4$

$36(65.5)$

$24(43.6)$

3 (5.5)

20 (36.4)

$14(25.5)$

4 (7.3)

$5(9.1)$

$30(54.5)$

$29(52.7)$

$11(20.0)$

$49(89.1)$

$6(10.9)$

$1(1.8)$

$1(1.8)$

$30(54.5)$

$0(0)$

$1(1.8)$

Data are presented as mean \pm standard deviation or $n(\%)$ among 55 subjects in the full analysis set. BMI, body mass index; DPP-4, dipeptidyl peptidase-4; SGLT2, sodium-glucose transport protein 2; GLP1, glucagon-like peptide-1. 


\section{Characteristics}

Insulin

Combination agent

Antihypertensive agent

Antithrombotic agent

Lipid-lowering agent

Antihyperuricemic agent

Other concomitant agents

Data are presented as mean \pm standard deviation or $n(\%)$ among 55 subjects in the full analysis set. BMI, body mass index; DPP-4, dipeptidyl peptidase-4; SGLT2, sodium-glucose transport protein 2; GLP1, glucagon-like peptide-1.

\section{mean \pm standard deviation or $n(\%)$}

$1(1.8)$

$13(23.6)$

$26(47.3)$

2 (3.6)

$22(40.0)$

$4(7.3)$

$36(65.5)$

\section{Primary endpoints}

The primary endpoints of this study were the change and percent change in AST, ALT, Y-GTP, and HbA1c levels from baseline to week 52 . The AST, ALT, $Y$-GTP, and HbA1c significantly decreased from baseline to week 52. The AST levels were 40.2 $\pm 16.4 \mathrm{IU} / \mathrm{L}$ and $28.0 \pm 13.6 \mathrm{IU} / \mathrm{L}$ at baseline and week 52 , respectively, and the change from baseline to week 52 was $-12.2 \pm 13.0 \mathrm{IU} / \mathrm{L}(P<0.0001)$. The ALT levels were $73.0 \pm$ $39.6 \mathrm{IU} / \mathrm{L}$ and $46.3 \pm 32.3 \mathrm{IU} / \mathrm{L}$ at baseline and week 52 , respectively, and the change from baseline to week 52 was $-25.2 \pm 23.9 \mathrm{IU} / \mathrm{L}(P<0.0001)$. The $\mathrm{\gamma}$-GTP levels were $78.2 \pm 38.8$ and $54.6 \pm 28.2 \mathrm{IU} / \mathrm{L}$ at baseline and week 52, respectively, and the change from baseline to week 52 was $-21.4 \pm 22.1 \mathrm{IU} / \mathrm{L}(P<$ 0.0001). The $\mathrm{HbA} 1 \mathrm{c}$ levels were $7.5 \% \pm 0.5 \%$ and $7.0 \pm 0.6 \%$ at baseline and week 52 , respectively, and the change from baseline to week 52 was $-0.6 \% \pm 0.5 \%(P<0.0001)$ (Table 2$)$. The AST, ALT, Y-GTP, and $\mathrm{HbA} 1 \mathrm{c}$ levels significantly decreased from baseline to weeks 12 and 24. The changes in AST level from baseline were $-8.9 \pm 11.1 \mathrm{IU} / \mathrm{L}(P<0.0001)$ and $-10.5 \pm 13.9 \mathrm{IU} / \mathrm{L}(P<0.0001)$ at weeks 12 and 24 , respectively. The changes in ALT level from baseline were - $17.5 \pm 19.4 \mathrm{IU} / \mathrm{L}(P<0.0001)$ and $-21.0 \pm$ $26.0 \mathrm{IU} / \mathrm{L}(P<0.0001)$ at weeks 12 and 24 , respectively. The changes in $\mathrm{y}$-GTP level from baseline were $12.5 \pm 19.1 \mathrm{IU} / \mathrm{L}(P<0.0001)$ and $-12.8 \pm 26.7 \mathrm{IU} / \mathrm{L}(P=0.0012)$ at weeks 12 and 24 , respectively. The changes in $\mathrm{HbA} 1 \mathrm{c}$ level from baseline were $-0.5 \% \pm 0.4 \%(P<0.0001)$ and $-0.5 \% \pm 0.5 \%(P<0.0001)$ at weeks 12 and 24, respectively. 
Table 2

Primary endpoints

\begin{tabular}{|c|c|c|c|c|c|c|c|c|c|}
\hline \multirow[t]{2}{*}{ Endpoint } & \multirow[t]{2}{*}{ Week } & \multirow[b]{2}{*}{$n$} & \multirow{2}{*}{$\begin{array}{l}\text { Measurement } \\
\text { mean } \pm \text { SD }\end{array}$} & \multicolumn{2}{|c|}{ Change } & \multicolumn{4}{|c|}{ Percent change } \\
\hline & & & & $n$ & $\begin{array}{l}\text { mean } \pm \\
\text { SD }\end{array}$ & $\begin{array}{l}p \\
\text { value }\end{array}$ & $n$ & $\begin{array}{l}\text { mean } \pm \\
\text { SD }\end{array}$ & $\begin{array}{l}p \\
\text { value }\end{array}$ \\
\hline \multirow[t]{4}{*}{ AST (IU/L) } & 0 & 55 & $40.2 \pm 16.4$ & & & & & & \\
\hline & 12 & 54 & $30.9 \pm 10.5$ & 54 & $\begin{array}{l}-8.9 \pm \\
11.1\end{array}$ & $\begin{array}{l}< \\
0.0001\end{array}$ & 54 & $\begin{array}{l}-17.5 \pm \\
23.6\end{array}$ & $<0.0001$ \\
\hline & 24 & 52 & $29.4 \pm 12.3$ & 52 & $\begin{array}{l}-10.5 \pm \\
13.9\end{array}$ & $\begin{array}{l}< \\
0.0001\end{array}$ & 52 & $\begin{array}{l}-19.7 \pm \\
34.3\end{array}$ & 0.0001 \\
\hline & 52 & 50 & $28.0 \pm 13.6$ & 50 & $\begin{array}{l}-12.2 \pm \\
13.0\end{array}$ & $\begin{array}{l}< \\
0.0001\end{array}$ & 50 & $\begin{array}{l}-26.1 \pm \\
24.8\end{array}$ & $<0.0001$ \\
\hline \multirow[t]{4}{*}{ ALT (IU/L) } & 0 & 55 & $73.0 \pm 39.6$ & & & & & & \\
\hline & 12 & 54 & $54.1 \pm 28.6$ & 54 & $\begin{array}{l}-17.5 \pm \\
19.4\end{array}$ & $\begin{array}{l}< \\
0.0001\end{array}$ & 54 & $\begin{array}{l}-19.4 \pm \\
25.3\end{array}$ & $\begin{array}{l}< \\
0.0001\end{array}$ \\
\hline & 24 & 52 & $49.7 \pm 30.0$ & 52 & $\begin{array}{l}-21.0 \pm \\
26.0\end{array}$ & $\begin{array}{l}< \\
0.0001\end{array}$ & 52 & $\begin{array}{l}-22.5 \pm \\
36.8\end{array}$ & $\begin{array}{l}< \\
0.0001\end{array}$ \\
\hline & 52 & 50 & $46.3 \pm 32.3$ & 50 & $\begin{array}{l}-25.2 \pm \\
23.9\end{array}$ & $\begin{array}{l}<.0001 \\
0.0\end{array}$ & 50 & $\begin{array}{l}-31.1 \pm \\
30.1\end{array}$ & $<0.0001$ \\
\hline \multirow{4}{*}{$\begin{array}{l}\text { Y-GTP } \\
(\mathrm{IU} / \mathrm{L})\end{array}$} & 0 & 55 & $78.2 \pm 38.8$ & & & & & & \\
\hline & 12 & 54 & $63.4 \pm 29.7$ & 54 & $\begin{array}{l}-12.5 \pm \\
19.1\end{array}$ & $\begin{array}{l}<.0001 \\
0.000\end{array}$ & 54 & $\begin{array}{l}-14.1 \pm \\
20.2\end{array}$ & $<0.0001$ \\
\hline & 24 & 52 & $62.8 \pm 36.1$ & 52 & $\begin{array}{l}-12.8 \pm \\
26.7\end{array}$ & 0.0012 & 52 & $\begin{array}{l}-15.5 \pm \\
27.0\end{array}$ & 0.0001 \\
\hline & 52 & 50 & $54.6 \pm 28.2$ & 50 & $\begin{array}{l}-21.4 \pm \\
22.1\end{array}$ & $\begin{array}{l}< \\
0.0001\end{array}$ & 50 & $\begin{array}{l}-26.0 \pm \\
24.5\end{array}$ & $<0.0001$ \\
\hline \multirow[t]{4}{*}{ HbA1c (\%) } & 0 & 55 & $7.5 \pm 0.5$ & & & & & & \\
\hline & 12 & 54 & $7.0 \pm 0.5$ & 54 & $-0.5 \pm 0.4$ & $\begin{array}{l}< \\
0.0001\end{array}$ & 54 & $-7.0 \pm 4.9$ & $\begin{array}{l}< \\
0.0001\end{array}$ \\
\hline & 24 & 52 & $7.0 \pm 0.5$ & 52 & $-0.5 \pm 0.5$ & $\begin{array}{l}<.0001 \\
0.000\end{array}$ & 52 & $-7.0 \pm 6.3$ & $\begin{array}{l}<.0001 \\
0.0\end{array}$ \\
\hline & 52 & 50 & $7.0 \pm 0.6$ & 50 & $-0.6 \pm 0.5$ & $\begin{array}{l}< \\
0.0001\end{array}$ & 50 & $-7.8 \pm 6.3$ & $\dot{0} 0001$ \\
\hline
\end{tabular}




\section{Vital signs}

Body weight, BMI, and waist circumstance significantly decreased from baseline to weeks 12, 24, and 52 . The body weights were $80.4 \pm 14.9 \mathrm{~kg}, 77.5 \pm 14.0 \mathrm{~kg}, 78.0 \pm 14.0 \mathrm{~kg}$, and $77.6 \pm 14.2 \mathrm{~kg}$ at baseline and weeks 12,24 , and 52 , respectively. The changes in body weight from baseline were $-1.9 \pm 1.3 \mathrm{~kg}(P<$ $0.0001),-2.6 \pm 1.8 \mathrm{~kg}(P<0.0001)$, and $-3.1 \pm 2.5 \mathrm{~kg}(P<0.0001)$ at weeks 12,24 , and 52 , respectively. The BMI were $29.2 \pm 4.0 \mathrm{~kg} / \mathrm{m}^{2}, 28.3 \pm 4.0 \mathrm{~kg} / \mathrm{m}^{2}, 28.3 \pm 3.8 \mathrm{~kg} / \mathrm{m}^{2}$, and $28.0 \pm 3.8 \mathrm{~kg} / \mathrm{m}^{2}$ at baseline and weeks 12,24 , and 52 , respectively. The changes in BMl from baseline were $-0.7 \pm 0.5 \mathrm{~kg} / \mathrm{m}^{2}(P<0.0001)$, $-0.9 \pm 0.7 \mathrm{~kg} / \mathrm{m}^{2}(P<0.0001)$, and $-1.1 \pm 0.9 \mathrm{~kg} / \mathrm{m}^{2}(P<0.0001)$ at weeks 12,24 , and 52 , respectively. The waist circumferences were $98.5 \pm 9.9 \mathrm{~cm}, 97.0 \pm 10.2 \mathrm{~cm}, 97.2 \pm 9.9 \mathrm{~cm}$, and $96.7 \pm 10.3 \mathrm{~cm}$ at baseline and weeks 12,24 , and 52 , respectively. The changes in waist circumference from baseline were $-1.6 \pm$ $3.0 \mathrm{~cm}(P=0.0003),-1.6 \pm 3.2 \mathrm{~cm}(P=0.0006)$, and $-2.4 \pm 3.2 \mathrm{~cm}(P<0.0001)$ at weeks 12,24 , and 52 , respectively (Table 3 ). In contrast, the systolic blood pressure significantly decreased only from baseline $(131.9 \pm 11.9 \mathrm{~mm} \mathrm{Hg})$ to week $12(127.4 \pm 11.6 \mathrm{~mm} \mathrm{Hg})$. The change in systolic blood pressure from baseline was $-4.4 \pm 13.0 \mathrm{mmHg}(P=0.0172)$ and significant decreases disappeared after week 24 . No significant changes were observed for diastolic blood pressure or pulse. 
Table 3

Vital signs

\begin{tabular}{|c|c|c|c|c|c|c|c|c|c|}
\hline \multirow[t]{2}{*}{ Endpoint } & \multirow[t]{2}{*}{ Week } & \multirow[b]{2}{*}{$n$} & \multirow{2}{*}{$\begin{array}{l}\text { Measurement } \\
\text { mean } \pm \text { SD }\end{array}$} & \multicolumn{2}{|c|}{ Change } & \multirow[b]{2}{*}{$\begin{array}{l}p \\
\text { value }\end{array}$} & \multicolumn{3}{|c|}{ Percent change } \\
\hline & & & & $n$ & $\begin{array}{l}\text { mean } \\
\pm S D\end{array}$ & & $n$ & $\begin{array}{l}\text { mean } \\
\pm S D\end{array}$ & $\begin{array}{l}p \\
\text { value }\end{array}$ \\
\hline \multirow[t]{4}{*}{ Body weight (kg) } & 0 & 55 & $80.4 \pm 14.9$ & & & & & & \\
\hline & 12 & 53 & $77.5 \pm 14.0$ & 53 & $\begin{array}{l}-1.9 \pm \\
1.3\end{array}$ & $\begin{array}{l}< \\
0.0001\end{array}$ & 53 & $\begin{array}{l}-2.4 \pm \\
1.7\end{array}$ & $\begin{array}{l}< \\
0.0001\end{array}$ \\
\hline & 24 & 52 & $78.0 \pm 14.0$ & 52 & $\begin{array}{l}-2.6 \pm \\
1.8\end{array}$ & $\begin{array}{l}< \\
0.0001\end{array}$ & 52 & $\begin{array}{l}-3.1 \pm \\
2.0\end{array}$ & $\begin{array}{l}< \\
0.0001\end{array}$ \\
\hline & 52 & 50 & $77.6 \pm 14.2$ & 50 & $\begin{array}{l}-3.1 \pm \\
2.5\end{array}$ & $\begin{array}{l}< \\
0.0001\end{array}$ & 50 & $\begin{array}{l}-3.8 \pm \\
2.9\end{array}$ & $\begin{array}{l}< \\
0.0001\end{array}$ \\
\hline \multirow[t]{4}{*}{$\mathrm{BMI}\left(\mathrm{kg} / \mathrm{m}^{2}\right)$} & 0 & 55 & $29.2 \pm 4.0$ & & & & & & \\
\hline & 12 & 53 & $28.3 \pm 4.0$ & 53 & $\begin{array}{l}-0.7 \pm \\
0.5\end{array}$ & $\begin{array}{l}<.0001 \\
0.000\end{array}$ & 53 & $\begin{array}{l}-2.4 \pm \\
1.7\end{array}$ & $\hat{0}_{0.0001}$ \\
\hline & 24 & 52 & $28.3 \pm 3.8$ & 52 & $\begin{array}{l}-0.9 \pm \\
0.7\end{array}$ & $\begin{array}{l}< \\
0.0001\end{array}$ & 52 & $\begin{array}{l}-3.1 \pm \\
2.0\end{array}$ & $\begin{array}{l}<.0001 \\
0\end{array}$ \\
\hline & 52 & 50 & $28.0 \pm 3.8$ & 50 & $\begin{array}{l}-1.1 \pm \\
0.9\end{array}$ & $\begin{array}{l}< \\
0.0001\end{array}$ & 50 & $\begin{array}{l}-3.8 \pm \\
2.9\end{array}$ & $\begin{array}{l}< \\
0.0001\end{array}$ \\
\hline
\end{tabular}

$\begin{array}{llll}\text { Waist } & 0 & 55 & 98.5 \pm 9.9\end{array}$

circumstance

$(\mathrm{cm})$

\begin{tabular}{|c|c|c|c|c|c|c|c|c|}
\hline 12 & 53 & $97.0 \pm 10.2$ & 53 & $\begin{array}{l}-1.6 \pm \\
3.0\end{array}$ & 0.0003 & 53 & $\begin{array}{l}-1.6 \pm \\
3.1\end{array}$ & 0.0004 \\
\hline 24 & 52 & $97.2 \pm 9.9$ & 52 & $\begin{array}{l}-1.6 \pm \\
3.2\end{array}$ & 0.0006 & 52 & $\begin{array}{l}-1.6 \pm \\
3.1\end{array}$ & 0.0005 \\
\hline 52 & 47 & $96.7 \pm 10.3$ & 47 & $\begin{array}{l}-2.4 \pm \\
3.2\end{array}$ & $\hat{0}_{0.0001}$ & 47 & $\begin{array}{l}-2.4 \pm \\
3.2\end{array}$ & $\hat{0}_{0.0001}$ \\
\hline
\end{tabular}

$\begin{array}{llll}\text { Systolic blood } \quad 0 & 55 & 131.9 \pm 11.9\end{array}$

pressure $(\mathrm{mm} \mathrm{Hg})$

$\begin{array}{lllllllll}12 & 53 & 127.4 \pm 11.6 & 53 & -4.4 \pm & 0.0172 & 53 & -2.9 \pm & 0.0383 \\ & & & & 13.0 & & & 9.8 & \\ 24 & 52 & 130.5 \pm 11.2 & 52 & \begin{array}{l}-1.0 \pm \\ 13.2\end{array} & 0.6019 & 52 & \begin{array}{l}-0.2 \pm \\ 10.0\end{array} & 0.8753 \\ & & & & & & \\ 52 & 50 & 129.8 \pm 12.5 & 50 & \begin{array}{c}-1.9 \pm \\ 14.6\end{array} & 0.3565 & 50 & \begin{array}{l}-0.9 \pm \\ 10.7\end{array} & 0.5373\end{array}$




\begin{tabular}{|c|c|c|c|c|c|c|c|c|c|}
\hline \multirow[t]{2}{*}{ Endpoint } & \multirow[t]{2}{*}{ Week } & \multicolumn{2}{|r|}{ Measurement } & \multicolumn{2}{|c|}{ Change } & \multicolumn{4}{|c|}{ Percent change } \\
\hline & & $n$ & mean $\pm S D$ & $n$ & $\begin{array}{l}\text { mean } \\
\pm S D\end{array}$ & $\begin{array}{l}p \\
\text { value }\end{array}$ & $n$ & $\begin{array}{l}\text { mean } \\
\pm S D\end{array}$ & $\begin{array}{l}p \\
\text { value }\end{array}$ \\
\hline \multirow{4}{*}{$\begin{array}{l}\text { Diastolic blood } \\
\text { pressure }(\mathrm{mm} \mathrm{Hg})\end{array}$} & 0 & 55 & $81.3 \pm 9.5$ & & & & & & \\
\hline & 12 & 53 & $79.2 \pm 8.8$ & 53 & $\begin{array}{l}-2.1 \pm \\
9.4\end{array}$ & 0.1178 & 53 & $\begin{array}{l}-1.8 \pm \\
11.9\end{array}$ & 0.2869 \\
\hline & 24 & 52 & $78.7 \pm 8.7$ & 52 & $\begin{array}{l}-2.4 \pm \\
9.4\end{array}$ & 0.0731 & 52 & $\begin{array}{l}-2.2 \pm \\
12.1\end{array}$ & 0.2077 \\
\hline & 52 & 50 & $79.8 \pm 8.5$ & 50 & $\begin{array}{l}-1.4 \pm \\
10.2\end{array}$ & 0.3223 & 50 & $\begin{array}{l}-0.81 \\
\pm 13.0\end{array}$ & 0.6629 \\
\hline \multirow[t]{4}{*}{ Pulse } & 0 & 53 & $75.0 \pm 10.7$ & & & & & & \\
\hline & 12 & 52 & $74.3 \pm 11.8$ & 50 & $\begin{array}{l}-0.3 \pm \\
9.7\end{array}$ & 0.8163 & 50 & $\begin{array}{l}0.15 \\
\pm 13.0\end{array}$ & 0.9371 \\
\hline & 24 & 52 & $73.7 \pm 11.6$ & 50 & $\begin{array}{l}-1.3 \pm \\
8.6\end{array}$ & 0.2809 & 50 & $\begin{array}{l}-1.35 \\
\pm 11.4\end{array}$ & 0.4096 \\
\hline & 52 & 48 & $73.8 \pm 12.0$ & 46 & $\begin{array}{l}-0.8 \pm \\
8.1\end{array}$ & 0.4930 & 46 & $\begin{array}{l}-0.77 \\
\pm 10.6\end{array}$ & 0.6219 \\
\hline
\end{tabular}

\section{Glucose metabolism}

The fasting plasma glucose levels significantly decreased from baseline to weeks 12,24 , and $52(148.8 \pm$ $20.7 \mathrm{mg} / \mathrm{dL}, 131.3 \pm 17.7 \mathrm{mg} / \mathrm{dL}, 133.2 \pm 23.5 \mathrm{mg} / \mathrm{dL}$, and $130.4 \pm 19.8 \mathrm{mg} / \mathrm{dL}$ at baseline and weeks 12 , 24 , and 52 , respectively. The changes in fasting plasma glucose level from baseline were $-17.9 \pm$ $15.5 \mathrm{mg} / \mathrm{dL}(P<0.0001),-16.8 \pm 20.2 \mathrm{mg} / \mathrm{dL}(P<0.0001)$, and $-18.8 \pm 19.5 \mathrm{mg} / \mathrm{dL}(P<0.0001)$ at weeks 12,24 , and 52 , respectively. Plasma insulin and HOMA-IR significantly decreased from baseline to weeks 12,24 , and 52. The plasma insulin levels were $12.8 \pm 7.0 \mu \mathrm{U} / \mathrm{mL}, 10.9 \pm 5.7 \mu \mathrm{U} / \mathrm{mL}, 11.0 \pm 5.8 \mu \mathrm{U} / \mathrm{mL}$, and $11.1 \pm 6.9 \mu \mathrm{U} / \mathrm{mL}$ at baseline and weeks 12,24 , and 52 , respectively. The changes in plasma insulin level from baseline were $-2.0 \pm 4.6 \mu \mathrm{U} / \mathrm{mL}(P=0.0026),-2.0 \pm 5.0 \mu \mathrm{U} / \mathrm{mL}(P=0.0065)$, and $-1.7 \pm 5.4 \mu \mathrm{U} / \mathrm{mL}$ $(P=0.0323)$ at weeks 12,24 , and 52 , respectively. The HOMA-IR were $4.8 \pm 2.7,3.6 \pm 2.1,3.7 \pm 2.2$, and 3.6 \pm 2.4 at baseline and weeks 12,24 , and 52 , respectively. The changes in HOMA-IR from baseline were $1.2 \pm 1.7(P<0.0001),-1.2 \pm 1.8(P<0.0001)$, and $-1.1 \pm 2.2(P=0.0007)$ at weeks 12 , 24 , and 52 , respectively. HOMA- $\beta$ did not significantly change (Table 4$)$. 
Table 4

Other efficacy endpoints

Endpoint
Week

Measurement

Change
Percent Change

$\begin{array}{lll}n & \text { mean } & p \\ \pm S D & \text { value }\end{array}$

Fasting plasma $\quad 0 \quad 55 \quad 148.8 \pm 20.7$

glucose $(\mathrm{mg} / \mathrm{dL})$

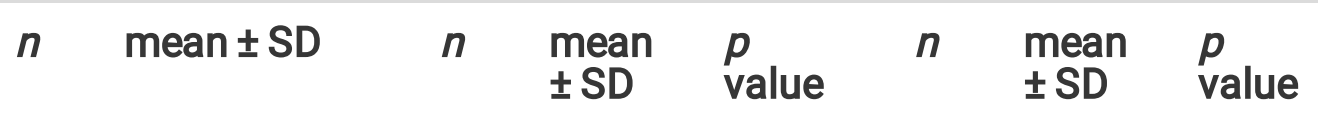

\begin{tabular}{|c|c|c|c|c|c|c|c|c|c|}
\hline & 12 & 54 & $131.3 \pm 17.7$ & 54 & $\begin{array}{l}-17.9 \\
\pm 15.5\end{array}$ & $<.0001$ & 54 & $\begin{array}{l}-11.4 \\
\pm 9.7\end{array}$ & $\begin{array}{l}<.0001 \\
0.001\end{array}$ \\
\hline & 24 & 52 & $133.2 \pm 23.5$ & 52 & $\begin{array}{l}-16.8 \\
\pm 20.2\end{array}$ & $\begin{array}{l}< \\
0.0001\end{array}$ & 52 & $\begin{array}{l}-10.8 \\
\pm 12.9\end{array}$ & $\begin{array}{l}< \\
0.0001\end{array}$ \\
\hline & 52 & 50 & $130.4 \pm 19.8$ & 50 & $\begin{array}{l}-18.8 \\
\pm 19.5\end{array}$ & $\begin{array}{l}<.0001 \\
\end{array}$ & 50 & $\begin{array}{l}-12.0 \\
\pm 12.6\end{array}$ & $\begin{array}{l}<.0001 \\
0.00\end{array}$ \\
\hline \multirow{4}{*}{$\begin{array}{l}\text { Plasma insulin } \\
(\mu \mathrm{U} / \mathrm{mL})\end{array}$} & 0 & 55 & $12.8 \pm 7.0$ & & & & & & \\
\hline & 12 & 53 & $10.9 \pm 5.7$ & 53 & $\begin{array}{l}-2.0 \pm \\
4.6\end{array}$ & 0.0026 & 53 & $\begin{array}{l}-11.5 \\
\pm 28.1\end{array}$ & 0.0043 \\
\hline & 24 & 52 & $11.0 \pm 5.8$ & 52 & $\begin{array}{l}-2.0 \pm \\
5.0\end{array}$ & 0.0065 & 52 & $\begin{array}{l}-10.1 \\
\pm 31.0\end{array}$ & 0.0230 \\
\hline & 52 & 49 & $11.1 \pm 6.9$ & 49 & $\begin{array}{l}-1.7 \pm \\
5.4\end{array}$ & 0.0323 & 49 & $\begin{array}{l}-8.9 \pm \\
41.4\end{array}$ & 0.1396 \\
\hline \multirow[t]{4}{*}{ HOMA- $\beta$ (\%) } & 0 & 55 & $55.6 \pm 30.1$ & & & & & & \\
\hline & 12 & 53 & $59.3 \pm 28.3$ & 53 & $\begin{array}{l}3.8 \pm \\
25.3\end{array}$ & 0.2802 & 53 & $\begin{array}{l}14.9 \\
\pm 47.2\end{array}$ & 0.0258 \\
\hline & 24 & 52 & $61.0 \pm 32.1$ & 52 & $\begin{array}{l}5.7 \pm \\
29.4\end{array}$ & 0.1710 & 52 & $\begin{array}{l}18.9 \\
\pm 54.9\end{array}$ & 0.0166 \\
\hline & 52 & 49 & $61.5 \pm 35.3$ & 49 & $\begin{array}{l}6.8 \pm \\
26.0\end{array}$ & 0.0714 & 49 & $\begin{array}{l}19.5 \\
\pm 55.4\end{array}$ & 0.0175 \\
\hline \multirow[t]{4}{*}{ HOMA-IR } & 0 & 55 & $4.8 \pm 2.7$ & & & & & & \\
\hline & 12 & 53 & $3.6 \pm 2.1$ & 53 & $\begin{array}{l}-1.2 \pm \\
1.7\end{array}$ & $\begin{array}{l}< \\
0.0001\end{array}$ & 53 & $\begin{array}{l}-21.9 \\
\pm 24.7\end{array}$ & $\begin{array}{l}< \\
0.0001\end{array}$ \\
\hline & 24 & 52 & $3.7 \pm 2.2$ & 52 & $\begin{array}{l}-1.2 \pm \\
1.8\end{array}$ & $\begin{array}{l}< \\
0.0001\end{array}$ & 52 & $\begin{array}{l}-20.0 \\
\pm 28.6\end{array}$ & $\begin{array}{l}< \\
0.0001\end{array}$ \\
\hline & 52 & 49 & $3.6 \pm 2.4$ & 49 & $\begin{array}{l}-1.1 \pm \\
2.2\end{array}$ & 0.0007 & 49 & $\begin{array}{l}-18.7 \\
\pm 41.3\end{array}$ & 0.0027 \\
\hline
\end{tabular}

Data are presented as $n$ and mean \pm standard deviation. HOMA- $\beta$, homeostatic model assessment beta; HOMA-IR, homeostatic model assessment of insulin resistance; FIB-4, fibrosis-4; FLI, fatty liver index; M2-BP, Mac-2 binding protein; hs-CRP, high-sensitivity C-reactive protein; IL-6, interleukin-6 


\begin{tabular}{|c|c|c|c|c|c|c|c|c|c|}
\hline \multirow[t]{2}{*}{ Endpoint } & \multirow[t]{2}{*}{ Week } & \multirow[b]{2}{*}{$n$} & \multirow{2}{*}{$\begin{array}{l}\text { Measurement } \\
\text { mean } \pm \text { SD }\end{array}$} & \multicolumn{3}{|c|}{ Change } & \multicolumn{3}{|c|}{ Percent Change } \\
\hline & & & & $n$ & $\begin{array}{l}\text { mean } \\
\pm S D\end{array}$ & $\begin{array}{l}p \\
\text { value }\end{array}$ & $n$ & $\begin{array}{l}\text { mean } \\
\pm S D\end{array}$ & $\begin{array}{l}p \\
\text { value }\end{array}$ \\
\hline \multirow[t]{3}{*}{ Ferritin (ng/mL) } & 0 & 55 & $191.4 \pm 144.9$ & & & & & & \\
\hline & 24 & 52 & $123.9 \pm 113.2$ & 52 & $\begin{array}{l}-64.6 \\
\pm 73.1\end{array}$ & $\begin{array}{l}< \\
0.0001\end{array}$ & 52 & $\begin{array}{l}-36.4 \\
\pm 25.1\end{array}$ & $\begin{array}{l}< \\
0.0001\end{array}$ \\
\hline & 52 & 49 & $113.6 \pm 97.9$ & 49 & $\begin{array}{l}-76.9 \\
\pm 74.2\end{array}$ & $\begin{array}{l}< \\
0.0001\end{array}$ & 49 & $\begin{array}{l}-40.1 \\
\pm 24.0\end{array}$ & $\begin{array}{l}< \\
0.0001\end{array}$ \\
\hline \multirow[t]{3}{*}{ M2-BP } & 0 & 55 & $0.74 \pm 0.30$ & & & & & & \\
\hline & 24 & 52 & $0.68 \pm 0.27$ & 52 & $\begin{array}{l}-0.06 \\
\pm 0.18\end{array}$ & 0.0187 & 52 & $\begin{array}{l}-5.5 \pm \\
20.5\end{array}$ & 0.0581 \\
\hline & 52 & 48 & $0.65 \pm 0.25$ & 48 & $\begin{array}{l}-0.08 \\
\pm 0.18\end{array}$ & 0.0027 & 48 & $\begin{array}{l}-8.3 \pm \\
22.1\end{array}$ & 0.0121 \\
\hline \multirow{4}{*}{$\begin{array}{l}\text { NAFLD fibrosis } \\
\text { score }\end{array}$} & 0 & 55 & $4.1 \pm 0.6$ & & & & & & \\
\hline & 12 & 54 & $4.0 \pm 0.8$ & 54 & $\begin{array}{l}-0.2 \pm \\
0.5\end{array}$ & 0.0225 & 54 & $\begin{array}{l}-3.8 \pm \\
12.3\end{array}$ & 0.0292 \\
\hline & 24 & 52 & $3.9 \pm 0.6$ & 52 & $\begin{array}{l}-0.5 \pm \\
0.4\end{array}$ & 0.0005 & 52 & $\begin{array}{l}-4.4 \pm \\
9.6\end{array}$ & 0.0019 \\
\hline & 52 & 50 & $4.0 \pm 0.7$ & 50 & $\begin{array}{l}-0.1 \pm \\
0.6\end{array}$ & 0.1178 & 50 & $\begin{array}{l}-2.9 \pm \\
14.6\end{array}$ & 0.1732 \\
\hline \multirow[t]{4}{*}{ FIB-4 index } & 0 & 55 & $3.1 \pm 1.8$ & & & & & & \\
\hline & 12 & 54 & $3.1 \pm 1.5$ & 54 & $\begin{array}{l}-0.1 \pm \\
0.9\end{array}$ & 0.6897 & 54 & $\begin{array}{l}3.1 \pm \\
25.0\end{array}$ & 0.3728 \\
\hline & 24 & 51 & $3.0 \pm 1.4$ & 51 & $\begin{array}{l}-0.1 \pm \\
0.8\end{array}$ & 0.3621 & 51 & $\begin{array}{l}2.0 \pm \\
21.6\end{array}$ & 0.5123 \\
\hline & 52 & 47 & $3.2 \pm 1.6$ & 47 & $\begin{array}{l}0.2 \pm \\
0.9\end{array}$ & 0.0774 & 47 & $\begin{array}{l}12.4 \\
\pm 25.8\end{array}$ & 0.0020 \\
\hline \multirow[t]{3}{*}{ FLI } & 0 & 55 & $73.3 \pm 16.7$ & & & & & & \\
\hline & 12 & 53 & $64.2 \pm 22.9$ & 53 & $\begin{array}{l}-8.9 \pm \\
13.6\end{array}$ & $\begin{array}{l}< \\
0.0001\end{array}$ & 53 & $\begin{array}{l}-13.6 \\
\pm 18.6\end{array}$ & $\begin{array}{l}< \\
0.0001\end{array}$ \\
\hline & 24 & 52 & $65.8 \pm 22.1$ & 52 & $\begin{array}{l}-7.5 \pm \\
10.3\end{array}$ & $\begin{array}{l}< \\
0.0001\end{array}$ & 52 & $\begin{array}{l}-11.8 \\
\pm 16.0\end{array}$ & $\begin{array}{l}<.0001 \\
0.0\end{array}$ \\
\hline
\end{tabular}

Data are presented as $n$ and mean \pm standard deviation. HOMA- $\beta$, homeostatic model assessment beta; HOMA-IR, homeostatic model assessment of insulin resistance; FIB-4, fibrosis-4; FLI, fatty liver index; M2-BP, Mac-2 binding protein; hs-CRP, high-sensitivity C-reactive protein; IL-6, interleukin-6 


\begin{tabular}{|c|c|c|c|c|c|c|c|c|c|}
\hline \multirow[t]{2}{*}{ Endpoint } & \multirow[t]{2}{*}{ Week } & \multicolumn{2}{|r|}{ Measurement } & \multicolumn{2}{|c|}{ Change } & \multicolumn{4}{|c|}{ Percent Change } \\
\hline & & $n$ & mean $\pm S D$ & $n$ & $\begin{array}{l}\text { mean } \\
\pm S D\end{array}$ & $\begin{array}{l}p \\
\text { value }\end{array}$ & $n$ & $\begin{array}{l}\text { mean } \\
\pm S D\end{array}$ & $\begin{array}{l}p \\
\text { value }\end{array}$ \\
\hline & 52 & 47 & $60.8 \pm 24.8$ & 47 & $\begin{array}{l}-12.5 \\
\pm 12.0\end{array}$ & $\begin{array}{l}<.0001 \\
0.000\end{array}$ & 47 & $\begin{array}{l}-20.1 \\
\pm 19.6\end{array}$ & $\begin{array}{l}< \\
0.0001\end{array}$ \\
\hline \multirow{3}{*}{$\begin{array}{l}\text { Type IV collagen } \\
\text { 7S domain } \\
\text { (ng/mL) }\end{array}$} & 0 & 55 & $4.6 \pm 1.2$ & & & & & & \\
\hline & 24 & 52 & $4.6 \pm 0.8$ & 52 & $\begin{array}{l}0.0 \pm \\
1.0\end{array}$ & 0.8387 & 52 & $\begin{array}{l}4.8 \pm \\
23.2\end{array}$ & 0.1423 \\
\hline & 52 & 49 & $4.6 \pm 0.7$ & 49 & $\begin{array}{l}0.1 \pm \\
1.0\end{array}$ & 0.4492 & 49 & $\begin{array}{l}6.3 \pm \\
24.5\end{array}$ & 0.0768 \\
\hline \multirow[t]{3}{*}{ hs-CRP (mg/dL) } & 0 & 55 & $0.11 \pm 0.13$ & & & & & & \\
\hline & 24 & 52 & $0.09 \pm 0.10$ & 52 & $\begin{array}{l}-0.02 \\
\pm 0.10\end{array}$ & 0.1222 & 52 & $\begin{array}{l}10.7 \\
\pm 96.3\end{array}$ & 0.4259 \\
\hline & 52 & 49 & $0.08 \pm 0.11$ & 49 & $\begin{array}{l}-0.03 \\
\pm 0.13\end{array}$ & 0.0968 & 49 & $\begin{array}{l}7.8 \pm \\
150.7\end{array}$ & 0.7172 \\
\hline \multirow[t]{2}{*}{ IL-6 (pg/mL) } & 0 & 55 & $2.1 \pm 2.7$ & & & & & & \\
\hline & 52 & 49 & $1.6 \pm 1.2$ & 49 & $\begin{array}{l}-0.3 \pm \\
1.1\end{array}$ & 0.0847 & 49 & $\begin{array}{l}-4.6 \pm \\
38.7\end{array}$ & 0.4062 \\
\hline $\begin{array}{l}\text { Data are presente } \\
\text { beta; HOMA-IR, ho } \\
\text { index; M2-BP, Ma }\end{array}$ & $\begin{array}{l}n \text { anc } \\
\text { ostatic }\end{array}$ & nean & $\begin{array}{l} \pm \text { standard devia } \\
\text { assessment of } \\
\text {; hs-CRP, high-se }\end{array}$ & & $\begin{array}{l}\text { DMA- } \beta \text {, } \\
\text { esistan } \\
\text { y C-reac }\end{array}$ & $\begin{array}{l}\text { meosta } \\
\text {; FIB-4, } \\
\text { ie protei }\end{array}$ & $\begin{array}{l}\text { mod } \\
\text { osis- } \\
\text { IL-6, }\end{array}$ & $\begin{array}{l}\text { ll assess } \\
\text { l; FLI, fat } \\
\text { tterleukir }\end{array}$ & $\begin{array}{l}\text { nent } \\
\text { y liver } \\
-6\end{array}$ \\
\hline
\end{tabular}

\section{Hepatic function biomarkers}

FLI is a surrogate liver fat marker and significantly decreased from baseline to weeks 24 and 52 . The FLI values were $73.3 \pm 16.7,64.2 \pm 22.9,65.8 \pm 22.1$, and $60.8 \pm 24.8$ at baseline and weeks 12,24 , and 52 , respectively. The changes in FLI from baseline were $-8.9 \pm 13.6(P<0.0001),-7.5 \pm 10.3(P<0.0001)$, and $-12.5 \pm 12.0(P<0.0001)$ at weeks 12,24 , and 52 , respectively. Ferritin is a hepatic impairment biomarker. Its levels significantly decreased from baseline to weeks 24 and 52 . The ferritin levels were $191.4 \pm$ $144.9 \mathrm{ng} / \mathrm{mL}, 123.9 \pm 113.2 \mathrm{ng} / \mathrm{mL}$, and $113.6 \pm 97.9 \mathrm{ng} / \mathrm{mL}$ at baseline and weeks 24 and 52 , respectively. The changes in ferritin level from baseline were $-64.6 \pm 73.1 \mathrm{ng} / \mathrm{mL}(P<0.0001)$ and -76.9 $\pm 74.2 \mathrm{ng} / \mathrm{mL}(P<0.0001)$ at weeks 24 and 52, respectively. The hepatic fibrosis biomarker level M2-BP decreased from baseline to weeks 24 and 52. The M2-BP levels were $0.74 \pm 0.30,0.68 \pm 0.27$, and $0.65 \pm$ 0.25 at baseline and weeks 24 and 52, respectively. The changes in M2-BP level from baseline were $0.06 \pm 0.18(P=0.0187)$ and $-0.08 \pm 0.18(P=0.0027)$ at weeks 24 and 52 , respectively. The NAFLD fibrosis score significantly decreased from baseline to weeks 12 and 24 . The NAFLD fibrosis scores were 
4.1 $\pm 0.6,4.0 \pm 0.8$ and $3.9 \pm 0.6$ at baseline and weeks 12 and 24 , respectively. The changes in NAFLD fibrosis score from baseline were $-0.2 \pm 0.5(P=0.0225)$ and $-0.5 \pm 0.4(P=0.0005)$ at weeks 12 and 24, respectively. However, there was no significant change in NAFLD fibrosis score by week 52 . The FIB-4 index and the type IV collagen $7 \mathrm{~S}$ domain did not significantly change from baseline (Table 4).

\section{Inflammation biomarkers}

The hs-CRP and IL-6 levels did not significantly change from baseline (Table 4).

\section{Safety outcomes}

Twenty-four adverse events were reported in eighteen out of fifty-five subjects (32.7\%) (Table 5). The most common adverse events were genital infection and dry skin (six subjects each; $10.9 \%$ ). Four serious adverse events (facial paresis, venous thrombosis, Báker's cyst, and gastric cancer) were reported during the study. Nevertheless, none of them was considered to be related to luseogliflozin administration. No hypoglycemia or severe hypoglycemia was reported during the study. 
Table 5

Adverse events

\begin{tabular}{|ll|}
\hline Adverse event & Frequency \\
\hline Death & $0(0)$ \\
\hline Any adverse events & $18(32.7)$ \\
\hline Serious adverse event & $4(7.3)$ \\
\hline Genital infection & $6(10.9)$ \\
\hline Dry skin & $6(10.9)$ \\
\hline Balanitis & $1(1.8)$ \\
\hline Dehydration & $1(1.8)$ \\
\hline Skin rash & $1(1.8)$ \\
\hline Constipation & $1(1.8)$ \\
\hline Genital itching & $1(1.8)$ \\
\hline Facial paresis & $1(1.8)$ \\
\hline Arthritis & $1(1.8)$ \\
\hline Disc herniation (Low back pain) & $1(1.8)$ \\
\hline Venous thrombosis & $1(1.8)$ \\
\hline Pulled muscle & $1(1.8)$ \\
\hline Baker cyst & $1(1.8)$ \\
\hline Gastric cancer & $1(1.8)$ \\
\hline Data are presented as $n$ (\%). & \\
\hline
\end{tabular}

\section{Discussion}

The purpose of this study was to assess the safety and efficacy of the SGLT2 inhibitor luseogliflozin in T2DM patients with hepatic dysfunction. We observed improvement in glycemic control and the hepatic function biomarkers AST, ALT, and Y-GTP. Moreover, FLI (a surrogate liver fat marker), ferritin (a hepatic impairment biomarker), M2-BP, and the NAFLD fibrosis score (hepatic fibrosis biomarkers) were significantly improved in response to luseogliflozin administration.

Several previous studies in T2DM patients showed that luseogliflozin administration decreased hepatic function biomarkers $[8,14,16]$. A recent single-arm LEAD trial in T2DM patients with NAFLD demonstrated a significant decrease in hepatic function biomarkers [17]. The results of the present study 
were consistent with those of previous reports. The present study confirmed that luseogliflozin improved hepatic function in T2DM patients with hepatic dysfunction and ALT levels $>31 \mathrm{IU} / \mathrm{L}$ at enrolment.

Additionally, this study revealed improvement in the hepatic fibrosis biomarkers M2-BP and NAFLD fibrosis score following luseogliflozin administration in T2DM patients with hepatic dysfunction. The LEAD trial showed decrease in the AST, ALT, Y-GTP, and ferritin levels in T2DM patients with NAFLD. However, the hepatic fibrosis markers FIB4 index, NAFLD fibrosis score, type IV collagen 7S, and M2BP were unchanged [17]. Hence, luseogliflozin may only be able to ameliorate mild to moderate hepatic dysfunction but not established NAFLD.

The beneficial effects of SGLT2 inhibitors on body weight and composition have been reported [18-21]. The present study demonstrated significant reduction in body weight, BMI, and waist circumstance after luseogliflozin administration. The surrogate liver fat marker FLI significantly decreased in the present study. This finding was consistent with that reported by previous studies which demonstrated decrease in liver fat following SGLT2 inhibitor treatment [22-24] and might explain the hepatic function improvement observed here.

This trial also disclosed that that plasma insulin levels and HOMA-IR were significantly decreased by luseogliflozin administration. This finding was consistent with earlier reports of reductions in HOMA-IR (insulin resistance improvement) by SGLT2 inhibitors $[25,26]$. In contrast, HOMA- $\beta$ did not significantly improve in the present study. SGLT2 inhibitors apparently improved pancreatic $\beta$-cell function in animal models [27]. However, no study has evaluated the effects of SGLT2 inhibitors on pancreatic $\beta$-cell function in humans. A Korean clinical study in Korea showed that a group responding well to SGLT2 inhibitors presented with relatively higher HOMA than the group responding poorly to SGLT2 inhibitors. Nevertheless, the HOMA- $\beta$ level was not associated with SGLT2 inhibitor responsiveness [28]. The results of this study suggested that SGLT2 inhibitors improved insulin resistance but not insulin secretion in the pancreatic $\beta$-cells of T2DM patients.

Here, luseogliflozin administration did not improve inflammation because it did not lower the hs-CRP or IL-6 levels. It has been previously reported that SGLT2 inhibitors suppress inflammation in animal models $[29,30]$. However, only a few human trials reported an association between SGLT2 inhibitors and inflammation [31, 32]. Further studies are needed to assess the efficacy of SGLT2 inhibitors in attenuation of inflammation.

No serious adverse events associated with luseogliflozin were detected in the present study. Genital infection and dry skin were the most commonly observed adverse reactions here but they are well-known side effects of SGLT2 inhibitors. There were no remarkable occurrences of any unknown side effects. No hypoglycemia was recorded during this trial. It was reported earlier that SGLT2 inhibitors lowered plasma glucose in an insulin-independent manner and posed a low risk of inducing hypoglycemia [33]. Moreover, recent large-scale clinical trials demonstrated that SGLT2 inhibitors prevented mortality, macrovascular complications, and renal impairment progression associated with T2DM $[9,10]$. Overall, SGLT2 inhibitors are well tolerated in T2DM patients with hepatic dysfunction. 
The study protocol described the enrolment of 50 subjects. Nevertheless, it was feasible for the Seino Internal Medical Clinic to support the enrolment of 55 subjects. The over-registration was reported to the Fukushima Medical University Certified Review Board and approved by them. The last five subjects were excluded and the results of the present study were re-analyzed with only 50 participants. Once again, all primary endpoints (ALT, AST, $\mathrm{Y}$-GTP, and HbA1c) showed significant improvements. Therefore, overregistration by five patients did not influence the outcome of this trial and all 55 subjects were included in the FAS and analyzed.

This study had several limitations. First, there were relatively few enrolled subjects in this trial and it was conducted in only one clinical institution (Seino Internal Medical Clinic) in Japan. Second, it was a singlearm trial. Third, the eligibility criterion hepatic dysfunction was defined only by the ALT level at enrolment. Therefore, the participants in this trial had mild to moderate hepatic dysfunction. These constraints may limit the generalizability of this study and large-scale, multicenter, randomized controlled trials are required to validate its findings.

\section{Conclusion}

Luseogliflozin administration was tolerated in T2DM patients with hepatic dysfunction. It improved hepatic function, reduced liver fat, and attenuated liver injury and fibrosis. The results of this trial might facilitate the establishment of a treatment strategy for T2DM patients with hepatic dysfunction caused by SGLT2 inhibitors.

\section{Abbreviations}

ALT alanine aminotransferase

AST aspartate aminotransferase

BMI body mass index

eGFR estimated glomerular filtration rate

FAS full analysis set

FIB-4 Fibrosis-4

FLI fatty liver index

FPG fasting plasma glucose

Y-GTP gamma-glutamyl transpeptidase

$\mathrm{HbA1c}$ hemoglobin A1c 
HOMA- $\beta$ homeostatic model assessment beta

HOMA-IR homeostasis model assessment of insulin resistance

hs-CRP high-sensitivity C-reactive protein

IL-6 interleukin-6

M2-BP Mac-2-binding protein

NAFLD nonalcoholic fatty liver disease

NASH nonalcoholic steatohepatitis

PPS per protocol set

SGLT2 sodium glucose co-transporter 2

T2DM type 2 diabetes mellitus

\section{Declarations}

\section{Ethics approval and consent to participate}

The study protocol was initially approved by the Institutional Review Board of the Seino Internal Medical Clinic in November 2016 according to the Ethical Guidelines for Medical and Health Research Involving Human Subjects issued by the Ministry of Health, Labour and Welfare in Japan. As the Clinical Trials Act was dispensed, the study protocol was reinspected and approved by the Fukushima Medical University Certified Review Board which obtained certification from the Minister of Health, Labour and Welfare in Japan in March 2019. The study was conducted in accordance with the Declaration of Helsinki, the Ethical Guidelines for Medical and Health Research Involving Human Subjects issued by the Ministry of Health, Labour and Welfare in Japan, the Clinical Trials Act, and other current legal regulations in Japan. Written informed consent was obtained from all participants after full explanation of the study.

\section{Consent for publication}

As this manuscript does not contain any individual personal data, consent for publication was not required or applicable.

\section{Availability of data and materials}

The datasets generated and/or analyzed during the current study are not publicly available as there is no third-party data sharing statement in the study protocol. Furthermore, we did not secure approval for sharing the informed consent documents from the Institutional Review Board of the Seino Internal Medical Clinic or the Fukushima Medical University Certified Review Board. 
Competing interests

HS received lecture fees from Shionogi Co. Ltd., Mitsubishi Tanabe Pharma Co., Astellas Pharma Inc., Novartis Pharma K.K., Eli Lilly and Co., Ono Pharmaceutical Co. Ltd., MSD K.K., and Sanofi K.K.

HS was awarded research grants from Taisho Pharmaceutical Co. Ltd., Takeda Pharmaceutical Co. Ltd., Novo Nordisk Pharma Ltd., Sanofi K.K., MSD K.K., Boehringer Ingelheim Pharmaceuticals, Inc., AstraZeneca K.K., Sanwa Kagaku Kenkyusho Co. Ltd., YL Biologics Ltd., and Japan Tobacco Inc.

\section{Funding}

This study was financially supported by Taisho Pharmaceutical Co. Ltd.

\section{Author contributions}

HS conceived and designed the study. HS enrolled the subjects, acquired the data, and drafted and revised the manuscript. HS met the International Committee of Medical Journal Editors (ICMJE) criteria for authorship of this article and assumed full responsibility for its overall integrity.

\section{Acknowledgments}

The author thanks all study participants and the clinical staff for their assistance in the execution of this trial. The author thanks Soiken Inc. for their support in writing the medical content of the manuscript.

\section{References}

1. Hamed AE, Elsahar M, Elwan NM, et al. Managing diabetes and liver disease association. Arab J Gastroenterol. 2018;19:166-79.

2. Hamed AE, Elwan N, Naguib M, et al. Diabetes association with liver diseases: An overview for clinicians. Endocr Metab Immune Disord Drug Targets. 2019;19:274-80.

3. Leite NC, Salles GF, Araujo AL, Villela-Nogueira CA, Cardoso CR. Prevalence and associated factors of non-alcoholic fatty liver disease in patients with type-2 diabetes mellitus. Liver Int. 2009;29:113-19.

4. Chan WK, Tan AT, Vethakkan SR, Tah PC, Vijayananthan A, Goh KL. Non-alcoholic fatty liver disease in diabetics-prevalence and predictive factors in a multiracial hospital clinic population in Malaysia. J Gastroenterol Hepatol. 2013;28:1375-83.

5. Tolman KG, Fonseca V, Dalpiaz A, Tan MH. Spectrum of liver disease in type 2 diabetes and management of patients with diabetes and liver disease. Diabetes Care. 2007;30:734-43.

6. Balkau B, Eschwege E, Ducimetiere P, Richard JL, Warnet JM. The high risk of death by alcohol related diseases in subjects diagnosed as diabetic and impaired glucose tolerant: the Paris Prospective Study after 15 years of follow-up. J Clin Epidemiol. 1991;44:465-74.

7. de Marco R, Locatelli F, Zoppini G, Verlato G, Bonora E, Muggeo M. Cause-specific mortality in type 2 diabetes. The Verona Diabetes Study. Diabetes Care. 1999;22:756-61. 
8. Seino Y, Inagaki N, Haneda M, et al. Efficacy and safety of luseogliflozin added to various oral antidiabetic drugs in Japanese patients with type 2 diabetes mellitus. J Diabetes Investig. 2015;6:443-53.

9. Zinman B, Wanner C, Lachin JM, et al. Empagliflozin, cardiovascular outcomes, and mortality in type 2 diabetes. N Engl J Med. 2015;373:2117-28.

10. Neal B, Perkovic V, Mahaffey KW, et al. Canagliflozin and cardiovascular and renal events in type 2 diabetes. N Engl J Med. 2017;377:644-57.

11. Seino Y, Sasaki T, Fukatsu A, Ubukata M, Sakai S, Samukawa Y. Efficacy and safety of luseogliflozin as monotherapy in Japanese patients with type 2 diabetes mellitus: a randomized, double-blind, placebo-controlled, phase 3 study. Curr Med Res Opin. 2014;30:1245-55.

12. Nishimura R, Osonoi T, Kanada S, et al. Effects of luseogliflozin, a sodium-glucose co-transporter 2 inhibitor, on 24-h glucose variability assessed by continuous glucose monitoring in Japanese patients with type 2 diabetes mellitus: A randomized, double-blind, placebo-controlled, crossover study. Diabetes Obes Metab. 2015;17:800-04.

13. Seino Y, Kaku K, Inagaki N, et al. Fifty-two-week long-term clinical study of luseogliflozin as monotherapy in Japanese patients with type 2 diabetes mellitus inadequately controlled with diet and exercise. Endocr J. 2015;62:593-603.

14. Sakai S, Kaku K, Seino Y, et al. Efficacy and safety of the SGLT2 inhibitor luseogliflozin in Japanese patients with type 2 diabetes mellitus stratified according to baseline body mass index: Pooled analysis of data from 52-week Phase III trials. Clin Ther. 2016;38:843-62.

15. Qiang S, Nakatsu Y, Seno Y, et al. Treatment with the SGLT2 inhibitor luseogliflozin improves nonalcoholic steatohepatitis in a rodent model with diabetes mellitus. Diabetol Metab Syndr. 2015;7:104.

16. Kusunoki M, Natsume Y, Sato D, et al. Luseogliflozin, A sodium glucose co-transporter 2 inhibitor, alleviates hepatic impairment in Japanese patients with type 2 diabetes. Drug Res (Stuttg). 2016;66:603-06.

17. Sumida $Y$, Murotani $K$, Saito M, et al. Effect of luseogliflozin on hepatic fat content in type 2 diabetes patients with non-alcoholic fatty liver disease: A prospective, single-arm trial (LEAD trial). Hepatol Res. 2019;49:64-71.

18. Nauck MA, Del Prato S, Meier JJ, et al. Dapagliflozin versus glipizide as add-on therapy in patients with type 2 diabetes who have inadequate glycemic control with metformin: a randomized, 52-week, double-blind, active-controlled noninferiority trial. Diabetes Care. 2011;34:2015-22.

19. Hirose S, Nakajima S, Iwahashi Y, Seo A, Takahashi T, Tamori Y. Impact of the 8-week administration of tofogliflozin for glycemic control and body composition in Japanese patients with type 2 diabetes mellitus. Intern Med. 2016;55:3239-45.

20. Tosaki T, Kamiya H, Himeno T, et al. Sodium-glucose co-transporter 2 inhibitors reduce the abdominal visceral fat area and may influence the renal function in patients with type 2 diabetes. Intern Med. 2017;56:597-604. 
21. Sugiyama $S$, Jinnouchi $H$, Kurinami $N$, et al. Dapagliflozin reduces fat mass without affecting muscle mass in type 2 diabetes. J Atheroscler Thromb. 2018;25:467-76.

22. Shibuya T, Fushimi N, Kawai M, et al. Luseogliflozin improves liver fat deposition compared to metformin in type 2 diabetes patients with non-alcoholic fatty liver disease: A prospective randomized controlled pilot study. Diabetes Obes Metab. 2018;20:438-42.

23. Kuchay MS, Krishan S, Mishra SK, et al. Effect of empagliflozin on liver fat in patients with type 2 diabetes and nonalcoholic fatty liver disease: A randomized controlled trial (E-LIFT trial). Diabetes Care. 2018;41:1801-08.

24. Latva-Rasku A, Honka MJ, Kullberg J, et al. The SGLT2 inhibitor dapagliflozin reduces liver fat but does not affect tissue insulin sensitivity: A randomized, double-blind, placebo-controlled study with 8week treatment in type 2 diabetes patients. Diabetes Care. 2019;42:931-37.

25. Aso Y, Kato K, Sakurai S, et al. Impact of dapagliflozin, an SGLT2 inhibitor, on serum levels of soluble dipeptidyl peptidase- 4 in patients with type 2 diabetes and non-alcoholic fatty liver disease. Int $\mathrm{J}$ Clin Pract. 2019;73:e13335.

26. Liao X, Wang X, Li H, et al. Sodium-Glucose Cotransporter 2 (SGLT2) Inhibitor increases circulating zinc-alpha2-glycoprotein levels in patients with type 2 diabetes. Sci Rep. 2016;6:32887.

27. Steven S, Oelze M, Hanf A, et al. The SGLT2 inhibitor empagliflozin improves the primary diabetic complications in ZDF rats. Redox Biol. 2017;13:370-85.

28. Hong AR, Koo BK, Kim SW, Yi KH, Moon MK. Efficacy and safety of sodium-glucose cotransporter-2 inhibitors in Korean patients with type 2 diabetes mellitus in real-world clinical practice. Diabetes Metab J. 2019;43:590-606.

29. Nakatsu Y, Kokubo H, Bumdelger B, et al. The SGLT2 inhibitor luseogliflozin rapidly normalizes aortic mRNA levels of inflammation-related but not lipid-metabolism-related genes and suppresses atherosclerosis in diabetic ApoE KO mice. Int J Mol Sci. 2017;18:e1704.

30. Jaikumkao K, Pongchaidecha A, Chueakula N, et al. Dapagliflozin, a sodium-glucose co-transporter-2 inhibitor, slows the progression of renal complications through the suppression of renal inflammation, endoplasmic reticulum stress and apoptosis in prediabetic rats. Diabetes Obes Metab. 2018;20:2617-26.

31. Bouchi R, Terashima M, Sasahara Y, et al. Luseogliflozin reduces epicardial fat accumulation in patients with type 2 diabetes: a pilot study. Cardiovasc Diabetol. 2017;16:32.

32. Bonnet F, Scheen AJ. Effects of SGLT2 inhibitors on systemic and tissue low-grade inflammation: The potential contribution to diabetes complications and cardiovascular disease. Diabetes Metab. 2018;44:457-64.

33. Ghosh RK, Ghosh SM, Chawla S, Jasdanwala SA. SGLT2 inhibitors: a new emerging therapeutic class in the treatment of type 2 diabetes mellitus. J Clin Pharmacol. 2012;52:457-63.

\section{Figures}


Fig. 1

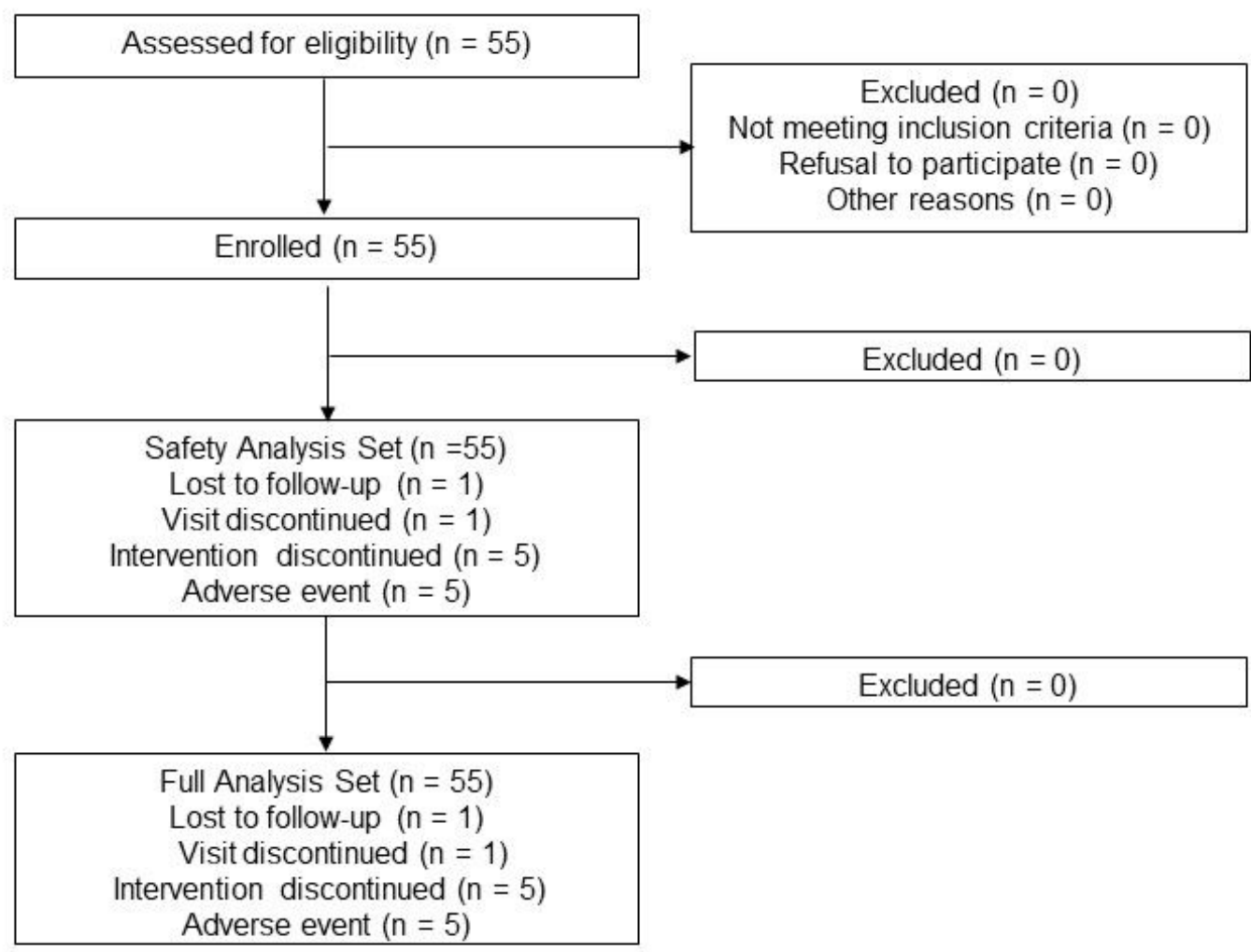

Figure 1

Flowchart depicting study participant enrolment, allocation, and analysis 55 eligible subjects were enrolled in this study. All 55 subjects were included and analyzed in the Safety Analysis Set and Full Analysis Set in this study. 